Mots. Les langages du politique

$96 \mid 2011$

Les discours politiques. Approches interactionnistes et multimodales

\title{
Sionisme. Oppositions militantes autour d'un terme à géométrie variable
}

\section{Marc Hecker}

\section{OpenEdition}

\section{Journals}

Édition électronique

URL : https://journals.openedition.org/mots/20299

DOI : $10.4000 /$ mots.20299

ISSN : 1960-6001

Éditeur

ENS Éditions

\section{Édition imprimée}

Date de publication : 5 septembre 2011

Pagination : $97-111$

ISBN : 978-2-84788-316-9

ISSN : 0243-6450

Référence électronique

Marc Hecker, «Sionisme. Oppositions militantes autour d'un terme à géométrie variable », Mots. Les

langages du politique [En ligne], 96 | 2011, mis en ligne le 05 septembre 2013, consulté le 22 avril 2022. URL : http://journals.openedition.org/mots/20299; DOI : https://doi.org/10.4000/mots.20299 


\section{Sionisme. Oppositions militantes autour d'un terme à géométrie variable}

Le conflit israélo-palestinien rejaillit, peut-être plus que nulle autre guerre, dans le domaine du langage. Selon les termes employés - Judée-Samarie ou Cisjordanie, implantations ou colonies, Arabes israéliens ou Palestiniens d'lsraël, barrière de sécurité ou mur de séparation, etc. -, un locuteur risque de se voir accuser d'utiliser une rhétorique partisane. Les plus fervents opposants à Israël refusent même de prononcer le nom de cet État, préférant le qualifier d' «entité sioniste».

Le terme sionisme est de ceux qui suscitent des discordes. Ce terme, apparu pour la première fois en 1890 sous la plume de Nathan Birnbaum¹ ${ }^{1}$, désigne un mouvement politique qui, dès la fin du $19^{\text {e }}$ siècle, prône le regroupement du peuple juif sur la terre d'Israël ou, pour reprendre la terminologie sioniste, le «retour à Sion». Cette définition demeure toutefois insuffisante. Elle élude notamment la question des divergences de vues existant au sein de la mouvance sioniste avant la naissance de l'État d'Israël et celle du devenir du sionisme après la création de cet État en 1948. Walter Laqueur note dans son Histoire du sionisme : "Selon une encyclopédie récente, le sionisme serait un mouvement politique mondial qui aurait été lancé par Théodore Herzl en 1897. Il serait tout aussi juste de dire que le socialisme fut fondé par Karl Marx en 1848. Il est manifestement difficile de rendre justice en une phrase aux créateurs de tout mouvement de quelque importance.» (Laqueur, 1973, p. 55)

De fait, des livres entiers ont été consacrés à la définition du sionisme. En 2007, par exemple, l'universitaire Denis Charbit a publié un ouvrage ayant pour titre Qu'est-ce que le sionisme ?2 Pour tenter de répondre à cette question, il analyse, entre autres, les textes fondateurs du mouvement sioniste à l’instar de

1. Sur Nathan Birnbaum, voir notamment Fishman, 1987. Voir aussi Kühntopf-Gentz, 1992, p. 118-139.

2. Charbit, 2007. D'autres ouvrages revenant sur la définition et sur l'histoire du sionisme ont été publiés récemment, parmi lesquels Greilsammer, 2005 et Bensoussan, 2001. En outre, plusieurs recueils de textes fondamentaux du sionisme existent, notamment Charbit, 1998 et Bénichou, 2008.

Institut français des relations internationales hecker@ifri.org 
Rome et Jérusalem de Moses Hess (1981), Autoémancipation de Léon Pinsker (2006) et bien sûr L'État juif de Théodore Herzl3.

Le but du présent article n'est pas de revenir en détail sur ces considérations qui relèvent davantage de l'histoire de la pensée politique. Il ne s'agit pas non plus de chercher à dégager une définition consensuelle du sionisme. L'objectif recherché consiste à montrer que le terme sionisme et ses dérivés - sioniste, antisionisme, post-sionisme, etc. - sont, en France, sources de tensions entre militants pro-israéliens et pro-palestiniens car ces deux sphères militantes en ont des interprétations bien différentes.

Pour ce faire, le sionisme sera tout d'abord présenté à travers un prisme pro-israélien en insistant sur le fait que la question de l'émigration vers Israël - l'alyah - continue à diviser. Un changement d'optique sera ensuite effectué en se plaçant du point de vue des militants pro-palestiniens pour qui le sionisme est connoté négativement et synonyme de colonialisme ou encore de racisme. Enfin, la question de l'antisionisme sera évoquée en montrant que ce terme nourrit lui aussi la discorde4.

\section{Alyah contre « sionisme de salon »}

Avant 1948, être « sioniste » signifiait simplement être favorable à la constitution d'un État juif ayant si possible Jérusalem pour capitale5. Cette question divisait jusqu'au sein de la communauté juive de France, comme en attestent les débats qui eurent lieu en 1943-1944 lorsqu'il fut question d'insérer une «clause sioniste » dans la première charte du Conseil représentatif des institutions juives de France (Crif) ${ }^{6}$. Depuis la création de l’État hébreu, la définition du terme sioniste fluctue davantage mais a toujours, du côté pro-israélien, une connotation positive. Certains se disent «sionistes » dans la mesure où ils sont pour le droit à l'existence d'Israël en tant qu'État juif. Dans un ouvrage collectif publié par l'Union des étudiants juifs de France (UEJF) et intitulé Le sionisme expliqué à nos potes, Paul Bernard, secrétaire national de l'association, explique ainsi :

Être sioniste, cela veut dire simplement être favorable à l'existence d'un État juif sur une partie de la Palestine mandataire : rien de moins mais rien de plus. Combien sont-ils, ceux qui sont sionistes sans le savoir, qui sont sincèrement convaincus de la légitimité de l'État d'Israël, mais qui se sentiraient outragés si on leur révélait quel adjectif sert à désigner cette conviction? (Bernard, 2003, p. 167)

3. Ce livre, publié initialement en 1896, a notamment été réédité en 2007 aux Éditions de L'Herne, et en 2008 , sous le titre L'État des juifs, à La Découverte.

4. Le terme «discorde» fait allusion à l'ouvrage de Rony Brauman et Alain Finkielkraut, 2006.

5. Théodore Herzl lui-même ne s'oppose pas à la constitution d'un État juif ailleurs qu'au ProcheOrient. Une partie de L'État juif s'intitule d'ailleurs «Palestine ou Argentine?» (Herzl, 2007, p. 58-62).

6. Voir à ce sujet Samuel Ghiles-Meilhac, 2009 et Catherine Nicault, 2001. 
Dans le même ouvrage, Patrick Klugman, alors président de l'UEJF, tient peu ou prou le même discours en affirmant :

Je suis sioniste. Je veux dire par là que je suis favorable à l'existence et au maintien de l’État juif institué dans la Palestine historique appelé «État d'Israël». Accepter la création d'un État juif, c'est d'abord reconnaître l'existence d'un peuple juif. (Klugman, 2003, p. 243)

Cette acception basse du terme sioniste n'induit pas d'appartenance religieuse particulière, la défense de l'existence de l'État d'Israël ne se limitant pas à la communauté juive. L'Association France-Israël en est l'illustration. Son ancien président, Michel Darmon, insiste sur la dimension non confessionnelle de cette organisation :

France-Israël n'est pas une association de la communauté juive. C'est l'association des Français amis d'Israël, quelle que soit leur tendance politique ou leur confession. C'est très important. Je suis le premier président qui soit juif depuis 1926, date de naissance de l'association.7

Gilles-William Goldnadel, successeur de Michel Darmon à la présidence de France-Israël, met lui aussi en avant la présence de nombreux non-juifs dans cette organisation, que ce soit dans les instances dirigeantes ou au niveau des membres de base ${ }^{8}$.

L'acception basse du terme sioniste n'est toutefois pas suffisante pour tous. Pour certains juifs français, être sioniste doit se traduire dans les faits par la «montée » vers Israël, l'alyah, et par l'acquisition de la citoyenneté israélienne en vertu de la «loi du retour»9. Plusieurs mouvements de jeunesse sionistes encouragent ouvertement l'immigration vers Israël. C'est le cas du Bétar, groupe se réclamant de la pensée de Vladimir Jabotinsky et proche du Likoud, principal parti de la droite israélienne. Un des responsables de ce groupe en France, Yaacov $\mathrm{X}^{10}$, présente clairement l'alyah comme un de ses objectifs essentiels :

Le but du mouvement est de faire partir les gens. À l'époque d'Arnaud [un ancien dirigeant], pratiquement toute la structure qu'il avait créée [est] partie. C'est quand même un peu moins d'une centaine qui sont montés en Israël. [...] Il y a vingt ans en France, lorsqu'on essayait de prôner l'alyah, lorsque je demandais à des jeunes, spontanément, "Qui compte faire son alyah?”, il y avait deux hurluberlus qui levaient la main. Sur cent personnes, la majorité disait : "On est sioniste, mais il ne faut quand même pas pousser." Mais aujourd'hui, j'ai posé la question à quelques jeunes que j'avais et toutes les mains se sont levées. J'étais vraiment épaté. ${ }^{11}$

7. Entretien avec Michel Darmon réalisé par l'auteur le 14 mai 2004.

8. Entretien avec Gilles-William Goldnadel réalisé par l'auteur le 10 juillet 2006.

9. Sur la «loi du retour», voir Abitbol, 2002.

10. À aucun moment, lors de la prise de contact puis de l'entretien, ce responsable du Bétar n'a donné son nom de famille.

11. Entretien avec Yaacov $X$ réalisé par l'auteur le 4 août 2006. 
Yaacov X a d'ailleurs lui-même fait son alyah. À la fin des années quatre-vingt et au début des années quatre-vingt-dix, il vivait en France et participait déjà aux activités du Bétar. Puis il a décidé de franchir le pas et de partir s'installer en Israël avant de revenir en France pour s'occuper de ce mouvement de jeunesse.

Le Bétar promeut l'alyah en organisant pour ses membres des voyages en Israël. Ces voyages sont courts et permettent aux jeunes de découvrir ce pays pendant leurs vacances. D’autres mouvements de jeunesse - comme le Bné Akiva, un groupe qui se définit comme sioniste et religieux - mettent en place des programmes plus longs pour favoriser l'alyah, connus sous le nom de archara. La archara permet à des jeunes qui ont pris la décision de s’installer en Israël de s’intégrer plus facilement. Voici comment le Bné Akiva présente ce programme :

De nos jours, nous, jeunes juifs français, avons l'avantage de nous voir proposer une large gamme de programmes pour parfaire notre intégration en Israël; car arrivé en Terre Sainte, cela constitue un objectif essentiel. Bourrés de rêves, de projets et d'idéaux, nous voulons faire partie intégrante de cette terre si longtemps désirée! Dès lors, une question évidente se pose à nous : quel est le programme idéal qui donnera un coup de pouce à mon intégration? Ce programme [la Archara] nous accompagne durant une année entière : il nous donne l'opportunité d'explorer Israël sous tous ses angles. ${ }^{12}$

Environ trois cents jeunes par an participeraient à des projets de ce type ${ }^{13}$. Un dirigeant du Bné Akiva, Raphaël Zaouch, explique : "Voilà près de 2000 ans que le peuple juif prie pour retourner en Israël. Voici maintenant 60 ans qu'a vu le jour l'État d'Israël et le retour du peuple juif sur sa terre. Je souhaite que nous puissions continuer ensemble cette ascension vers la Terre Sainte. »14

Entre 1500 et 3000 juifs de France effectuent leur alyah chaque année ${ }^{15}$. En 2007, les olim hadashim - expression employée en hébreu pour désigner les personnes ayant fait leur alyah récemment - provenant de France étaient 2659 , ce qui les situait en quatrième position derrière les pays de l'ex-URSS (6445 personnes), l’Éthiopie (3 607) et les États-Unis (2 957) ${ }^{16}$. Certaines personnes ne voient pas d'un très bon œil les juifs de diaspora qui se disent sio-

12. Cette présentation de la Archara figurait sur le site Internet du Bné Akiva que nous avions consulté le 16 février 2009. Le site [http://www.bneakiva.net/archara.htm] existe toujours mais cette page a disparu.

13. Source : brochure intitulée Mahanot Bné Akiva été 2008.

14. Raphaël Zaouch, « Lettre du chaliah », Mahanot Bné Akiva été 2008.

15. Les chiffres varient selon les années et selon les sources. Certains n'hésitent pas à parler de "guerre des chiffres». Voir Caroll Azoulay, "L'alya de France se place au-dessus de la mêlée», Guysen International News, 8 janvier 2008 [http://www.guysen.com/article_L-alya-de-France-seplace-au-dessus-de-la-melee_6582.html]. Le bureau central des statistiques, l'équivalent israélien de l'INSEE, donne des chiffres qui se situent plutôt dans le bas de la fourchette. Voir «Immigrants by period of immigration, country of birth and last country of residence », Central Bureau of Statistics, Statistical Abstract of Israel, 2008, tableau 4.4 [http://www1.cbs.gov.il/reader/shnaton/templ_shnaton_e.html?num_tab=sto4_04\&CYear=2008]. Sites consultés le 16 février 2009.

16. Stéphanie Zenati, "L'aliya en chute libre», Guysen International News, 24 décembre 2007 [http://www.guysen.com/tri_cat_gen.php?cat=\&page=331], consulté le 16 février 2009 . 
nistes mais refusent de franchir le pas de l'alyah. Ces derniers sont parfois qualifiés de «sionistes de salon »17 ou de «théoriciens». Théo Klein - ancien président du Conseil représentatif des institutions juives de France (Crif) qui a lui-même la double nationalité française et israélienne - explique par exemple : "Un vrai sioniste, c'est quelqu'un qui est assis face au mont Sion, à Jérusalem. Les autres sont des théoriciens. »18

Parmi les juifs de France qui n'ont pas souhaité faire leur alyah se trouve Emmanuel Weintraub, ancien vice-président du Crif. Pour lui, le fait d'être sioniste s'est notamment traduit par l'apprentissage de l'hébreu. Il raconte une anecdote à ce sujet :

Je sais l'hébreu, je le pratique et j'ai parfois des réactions étranges des Israéliens. C'est une chose qui leur paraît un peu suspecte. Par exemple, en Israël, quand il m'arrive à l'entrée ou à la sortie [du pays] de dire quelques mots à l'agent de sécurité, ce n'est pas une bonne idée parce que j'ai un passeport français et alors il me dit : "Où est votre passeport israélien?". Je dis : "Je n’en ai pas". Puis il me dit : "Alors, pourquoi tu parles hébreu?". II faut alors faire un grand discours sioniste pour expliquer pourquoi on parle hébreu. ${ }^{19}$

Un autre dirigeant de la communauté juive de France, Joël Mergui, président du Consistoire central20, clame haut et fort le droit des Français de confession juive à se déclarer sionistes et à défendre Israël sans nécessairement envisager de faire leur alyah. À la veille des élections européennes de 2009, pour protester contre la présence d'une «liste antisioniste», il a diffusé un message intitulé «Je suis affirma-sioniste». Plein d'enthousiasme, il expliquait:

Être affirma-sioniste, c'est affirmer que, sans la moindre incompatibilité, [...] on peut être citoyen français et soutenir Israël. Parce que c'est une démocratie pleine de vitalité et d'humanité, parce que c'est un pays à la pointe de la recherche médicale, scientifique et technologique, parce que le cinéma israélien est de haut niveau ou pour mille autres raisons. ${ }^{21}$

Si le terme sionisme est connoté positivement au sein des cercles pro-israéliens, il suscite au contraire une réelle méfiance, voire un rejet catégorique dans la mouvance pro-palestinienne.

17. Cette expression est revenue à deux reprises lors de discussions informelles qui ont eu lieu en Israël en mars 2008. Par ailleurs, Richard Wagman, président de l’Union juive française pour la paix, l'a employée lors d'un entretien réalisé par l'auteur le 13 avril 2006. L'expression «sioniste de salon » est aussi employée sur le site de la Ligue de défense juive dans un article très critique à l'égard de Kadima-France. Voir Élie Kling, "La techouva d’Alex Moïse », 7 novembre 2007 [http://www.liguededefensejuive.net/spip.php?article35], consulté le 23 février 2009.

18. Voir le documentaire de Stéphane Khémis et Jean-Baptiste Frappat intitulé «Théo Klein, juif, français, israélien » diffusé sur France 5 le $1^{\text {er }}$ octobre 2007.

19. Entretien avec Emmanuel Weintraub réalisé par l'auteur le 6 mai 2004.

20. Le Consistoire central est une institution créée à l'époque napoléonienne et qui a pour but d'organiser le culte juif en France. Voir notamment Lustman et al., 2004.

21. Joël Mergui, «Je suis affirma-sioniste», message envoyé par courrier électronique à tous les destinataires de la newsletter du Consistoire central le 5 juin 2009. 


\section{Sionisme, colonialisme et racisme}

La grande majorité des militants pro-palestiniens de France est pour une solution à deux États, c'est-à-dire un État palestinien créé à côté d'Israël avec la Ligne verte pour frontière. Si l'on considère que toute personne favorable au maintien de l'État d'Israël est sioniste, alors la grande majorité des propalestiniens de France serait sioniste. Pourtant, aucun militant pro-palestinien interviewé pour cette recherche ne s'est dit «sioniste». Quant au slogan «sioniste et pro-palestinien »22 utilisé par l'Union des étudiants juifs de France pendant la deuxième Intifada, il n'a été repris à son compte par aucune association pro-palestinienne, même parmi les plus modérées. Ceci s'explique par le fait que le sionisme est historiquement honni par les défenseurs des Palestiniens, qui l'associent au colonialisme et au racisme (Finkielkraut, 1983, p. 63).

Le discours de certains militants pro-palestiniens - notamment une partie de ceux qui militaient déjà pour cette cause avant la fin des années quatrevingt - reste marqué par quelques grands textes des années soixante. Au cours d'un entretien réalisé avec Walid Atallah, responsable du Mouvement de soutien à la résistance du peuple palestinien - une association particulièrement radicale qui s'oppose à l'existence de l'État d'Israël et soutient le retour des réfugiés palestiniens sur tout le territoire de la Palestine mandataire -, celui-ci a expliqué : «Pour nous, la lutte première, c'est contre le sionisme [...], contre l’idéologie coloniale sioniste qui est représentée par l'État d'Israël. »23 Pour expliquer sa position, il a mentionné, entre autres, la charte de l'OLP qui - à ses yeux - serait toujours valable dans sa version d'origine, malgré la déclaration de caducité faite par Yasser Arafat en 1989 et les modifications apportées par le Conseil national palestinien en 1996. Cette charte, rédigée en 1964 puis modifiée en 1968, est une véritable charge contre le sionisme. Sa «clé de voûte » 24 est l'article 22 qui débute par les phrases suivantes :

Le sionisme est un mouvement politique organiquement lié à l'impérialisme international et opposé à toute action de libération et à tout mouvement progressiste dans le monde. Il est raciste et fanatique par nature, agressif, expansionniste et colonial dans ses buts, et fasciste par ses méthodes. ${ }^{25}$

Un autre texte datant des années soixante et établissant un lien consubstantiel entre sionisme et colonialisme est encore régulièrement cité par les mili-

22. Voir notamment Klugman, 2003.

23. Entretien avec Walid Atallah réalisé par l'auteur le 16 août 2006.

24. L'expression «clé de voûte » est employée par Georges Élia Sarfati, 1997.

25. Le texte complet de la version de 1968 de la charte nationale palestinienne est en ligne [http:// perspective.usherbrooke.ca/bilan/servlet/BMDictionnaire?iddictionnaire=1443]. Site Internet de l'université de Sherbrooke consulté le 15 novembre 2009. 
tants pro-palestiniens de France. Il s'agit de l'article de Maxime Rodinson intitulé «Israël, fait colonial », écrit juste avant la guerre des Six jours et publié dans le numéro des Temps modernes paru en juin 1967. Au terme de soixantedix pages de considérations historiques sur l'origine du sionisme, le célèbre orientaliste conclut :

La formation de l'État d'Israël sur la terre palestinienne est l'aboutissement d'un processus qui s'insère parfaitement dans le grand mouvement d'expansion européo-américain des $19^{\mathrm{e}}$ et $20^{\mathrm{e}}$ siècles pour peupler ou dominer économiquement et politiquement les autres peuples. [...] Pour ce qui est des termes, il me semble que celui de processus colonial convient fort bien. (Rodinson, 1967, p. 83) S'il y a en effet une haine qui souvent dépasse la mesure, si les gouvernants et les idéologues construisent des mythes mobilisateurs autour du fait palestinien, c'est sur la base d'une donnée objective dont les dirigeants sionistes sont responsables, la colonisation d'une terre étrangère. (Ibid., p. 86)

Quarante ans après la publication de l'article de Maxime Rodinson, Bernard Ravenel, président de l'Association France Palestine Solidarité (AFPS) - une des principales associations françaises de soutien à la cause palestinienne -, a tenu à lui rendre hommage, qualifiant le texte paru dans les Temps modernes d' «article-événement» et le comparant, en termes d'impact, au «J'accuse» d’Émile Zola. Bernard Ravenel conclut :

À partir de l'article des Temps Modernes, Maxime Rodinson [...] aura été le grand penseur en France de la question palestinienne en même temps que de la question juive israélienne. Il aura fixé pour longtemps le cadre qui permet de penser les conditions théoriques d'une solution politique possible et souhaitable du conflit israélo-palestinien. (Ravenel, 2007)

Ainsi, la rhétorique associant sionisme et colonialisme fait aujourd'hui figure de lieu commun dans la sphère pro-palestinienne. Un groupe comme les «Indigènes de la République », de plus en plus visible dans les manifestations propalestiniennes, explique : «Le Mouvement des indigènes de la république (MIR) [...] constitue un espace d'organisation autonome de tous ceux qui veulent s'engager dans le combat contre les inégalités raciales qui cantonnent les Noirs, les Arabes et les musulmans à un statut analogue à celui des indigènes dans les anciennes colonies [...]. Plus généralement, le MIR lutte contre toutes les formes de domination impériale, coloniale et sioniste qui fondent la suprématie blanche à l'échelle internationale. „26 Le 8 mai 2008 , les Indigènes de la République faisaient partie des organisateurs de la «marche décoloniale » à Paris. Cette marche visait à protester contre la politique de Nicolas Sarkozy, qualifiée de « racisme républicain ». Cette politique se placerait « dans

26. Site du mouvement [http://www.indigenes-republique.fr/statique?id_article=189] consulté le $1^{\text {er }}$ mars 2009. 
la continuité coloniale à la française »; parmi les reproches faits au gouvernement figurait le «soutien sans faille à la politique guerrière des États-Unis et au colonialisme sioniste d'Israël $» 27$. Autre illustration, lors des manifestations pro-palestiniennes de janvier 2009 à Paris, les militants de la Confédération nationale du travail (CNT) défilaient avec une banderole arborant le slogan : "Le sionisme, c'est la guerre coloniale, le nettoyage ethnique »28.

La rhétorique liant sionisme et racisme existe aussi depuis longtemps et constitue en quelque sorte le prolongement logique du binôme sionisme-colonialisme. La résolution 3379 de l'Assemblée générale des Nations unies du 10 novembre 1975 énonçant : «Le sionisme est une forme de racisme et de discrimination raciale » a contribué à la diffusion - et d'une certaine manière à la légitimation - de ce discours. Cette résolution a été adoptée par 72 voix contre 35 et 32 abstentions avant d'être révoquée le 16 décembre 1991 par la résolution 46/86. La volonté d'assimiler le sionisme au racisme a connu de nouveaux développements pendant la deuxième Intifada, au moment notamment de la «Conférence mondiale contre le racisme, la discrimination raciale, la xénophobie et l'intolérance qui y est associée „29 tenue à Durban en septembre 2001. Avant même le début de la conférence, certains documents préparatoires assimilant le sionisme au racisme circulaient et le département d’État américain annonçait en conséquence que « le Secrétaire d’État américain ne se rendra[it] pas à Durban en raison du caractère anti-israélien du programme et de certains documents préparatoires ${ }^{30}$. Mary Robinson, haut-commissaire des Nations Unies aux droits de l'homme et secrétaire générale de la Conférence de Durban, tentait d'apaiser les esprits en déclarant : "Il est clair que la formulation "sionisme égale racisme" a été abandonnée dans les textes et que les délégations sont tombées d'accord pour qu'elle ne figure pas à l'ordre du jour. "31 Malgré ces déclarations, l'association du sionisme et du racisme s'est retrouvée au cœur des travaux du forum des ONG qui réunissait environ 3000 associations du monde entier. À la suite de débats houleux, une déclaration finale accusant Israël de «perpétration systématique de crimes racistes, dont des crimes de guerre, d'actes de génocide et de nettoyage ethnique «32 était adoptée tandis que certains documents échangés

27. Ce sont les termes employés dans l'appel à manifester diffusé le 14 février 2008 sur le site du mouvement [http://www.indigenes-republique.fr/article.php3?id_article=68], consulté le $1^{\mathrm{er}}$ mars 2009.

28. Observation de la manifestation pro-palestinienne du 10 janvier 2009 à Paris.

29. C'est l'intitulé officiel de cette conférence, plus connue sous l'appellation «Conférence de Durban ».

30. Annonce reprise dans une dépêche de l'Agence Europe du 31 août 2001, intitulée «La conférence mondiale contre le racisme s'ouvre sur fond de polémique sur le sionisme et l'indemnisation des victimes de l'esclavage».

31. La déclaration de Mary Robinson est citée dans une dépêche de l'Agence Europe du 31 août 2001 intitulée : «La conférence mondiale contre le racisme s'ouvre sur fond de polémique sur le sionisme et l'indemnisation des victimes de l'esclavage ».

32. Cette déclaration est citée dans une dépêche de l'Agence Reuters du 2 septembre 2001 intitulée : «3 000 ONG traitent Israël d’État raciste». 
appelaient ouvertement à un retour à la résolution 337933. L'adoption de cette déclaration finale a provoqué de nombreux remous et engendré le départ des délégations israélienne et américaine34. La conférence de Durban s'est finalement conclue par l'adoption laborieuse d'une «résolution de compromis », alors que la France et l'Union européenne avaient menacé de se retirer de la conférence si sa déclaration finale assimilait le sionisme à une forme de racisme35.

Sionisme, colonialisme et racisme se trouvent réunis dans la rhétorique comparant la situation proche-orientale à la période de l'apartheid en Afrique du Sud. Le discours associant la politique israélienne à une forme d'apartheid est très fréquent dans la mouvance pro-palestinienne, des ouvrages comme Palestine-Israël: la paix ou l'apartheid de Marwan Bishara (2002) ou Palestine: la paix, pas l'apartheid de Jimmy Carter (2007) étant régulièrement cités par les militants et vendus lors d'événements organisés par des associations de soutien à la cause palestinienne. Les propos liant sionisme et apartheid sont plus rares mais existent, comme en témoigne l'appel lancé par l'Union générale des étudiants de Palestine (Gups) pour participer à la «marche décoloniale » du 8 mai 2008. Cet appel stipule :

Bien loin de reconnaître sa responsabilité, Israël cherche à légitimer sa politique menée depuis 60 ans en nous faisant accepter l'idée qu'il est un État juif pour les juifs. Qu'adviendra-t-il des 1,5 millions de Palestiniens vivant encore sur leur terre, à l'intérieur des frontières de l'État colonial? Sont-ils définitivement devenus des étrangers chez eux? Et qu'adviendra-t-il du droit au retour des réfugiés? Ce nouvel apartheid que prône la doctrine sioniste est incompatible avec le droit et contraire aux valeurs que nous défendons. 36

Si le terme sionisme revêt donc historiquement une charge extrêmement négative au sein de la mouvance pro-palestinienne, les militants ne se disent pas tous, pour autant, "antisionistes». Ils insistent toutefois sur la nécessité de bien distinguer l'antisionisme de l'antisémitisme.

\section{Antisionisme et antisémitisme}

Un consensus existe parmi les principales associations pro-palestiniennes pour affirmer que l'antisionisme peut être légitime tandis que l'antisémitisme

33. De nombreux textes produits par le forum des ONG sont disponibles sur le site Internet de l'université de Dayton. Voir en particulier la section consacrée aux Palestiniens, où est mentionnée explicitement la résolution 3379 [http://academic.udayton.edu/race/o6hrights/WCAR2001/ NGOFORUM/Palestinans.htm]. Site consulté le 18 février 2009.

34. Ed Stoddard, «Racisme. Mise en garde française à Durban », Reuters, 5 septembre 2001.

35. L'expression « résolution de compromis » est employée dans une dépêche de l'Agence Reuters du 8 septembre 2001 intitulée : Jospin satisfait par la déclaration de Durban».

36. L'appel de la Gups a été relayé sur le site Internet des Indigènes de la République [http://www. indigenes-republique.fr/article.php3?id_article=64], consulté le 1er mars 2009. 
ne l'est pas. Les militants pro-palestiniens s'opposent donc à toute tentative d'amalgame entre ces deux notions. Pendant la deuxième Intifada, plusieurs ouvrages ont été publiés pour affirmer le droit de critiquer la politique israélienne - ou même éventuellement le sionisme - sans être nécessairement taxé d'antisémitisme. Dans Est-il permis de critiquer Israël?, Pascal Boniface dénonce l'instrumentalisation de l'antisémitisme :

Il ne faut pas nier l'antisémitisme. Il faut le combattre encore et toujours parce qu'il n'a pas disparu. Mais il ne faut pas non plus l'instrumentaliser. C'est ce que fait parfois le gouvernement israélien lorsqu'il se sent en difficulté face à la communauté internationale. (Boniface, 2003)

Quant à Denis Sieffert, il dénonce, dans un ouvrage collectif intitulé Antisémitisme, l'intolérable chantage, l'assimilation de «toute prise de position critique envers Israël à une forme d'antisémitisme» (Sieffert, 2003, p.18). Il ajoute : «C'est bien l'équation “Sharon égale sionisme égale judaïsme” qui est posée ici. » (Ibid.)

Il est vrai que la hausse spectaculaire des actes antisémites constatée en France à la fin de l'année 2000 et au printemps 200237 conduit certains responsables de la communauté juive à dénoncer le passage possible de l'antisionisme à l'antisémitisme ${ }^{38}$. Roger Cukierman, alors président du Crif, affirme par exemple sans détour :

On voit bien qu'à partir du moment où l'Intifada entraîne des actes violents anti-juifs, cela veut bien dire que le grand public français et en tout cas les gens concernés mélangent les deux. Je considère, pour ma part, que l'antisionisme est la nouvelle forme de l'antisémitisme. 39

Le même Roger Cukierman, dans son discours prononcé lors de l'édition 2003 du dîner du Crif, déclare :

Une connivence bizarre, une union contre nature entre la gauche laïque et révolutionnaire et les mouvements pro-palestiniens conduit à une application inattendue, le boycott. Une coordination appelle depuis le printemps dernier au boycott des produits israéliens. On a même vu des entreprises dites sionistes c'est-à-dire appartenant ou dirigées par des Français juifs, signalées à la vindicte du consommateur. Cela confirme, s'il en est besoin, combien l'antisionisme est le nouvel habit de l'antisémitisme. 40

37. Sur la hausse des actes antisémites pendant la deuxième Intifada, voir Wieviorka éd., 2005.

38. La rhétorique sur le passage de l'antisionisme à l'antisémitisme développée au début de la deuxième Intifada n'est pas sans rappeler les travaux de Léon Poliakov à la fin des années soixante. Voir en particulier Poliakov, 1969. À l'époque déjà, les militants pro-palestiniens s'indignaient contre l’instrumentalisation de l'antisémitisme. Voir par exemple Liebman, 1970.

39. Roger Cukierman est cité par Jean-Pierre Allali, 2002, p. 103.

40. Roger Cukierman, discours prononcé lors du dîner du Crif du 25 janvier 2003, en ligne [http:// www.crif.org/?page=articles_display/detail\&aid=659\&artyd=56], consulté le 19 février 2009 . 
La rhétorique sur le glissement de l'antisionisme à l'antisémitisme est par ailleurs développée dans plusieurs ouvrages, cités régulièrement par les militants pro-israéliens dont Les territoires perdus de la République (Brenner, 2004, p. 344 et 353), La nouvelle judéophobie (Taguieff, 2002, p. 74 et 187) ou encore Le nouveau bréviaire de la haine (Goldnadel, 2001). Ce dernier, dont le titre s'inspire du Bréviaire de la haine de Léon Poliakov (1951) a été écrit par l'avocat Gilles-William Goldnadel qui est devenu par la suite président de l'Association France-Israël. En 2002, l'Union des étudiants juifs de France et SOS Racisme publient ensemble un ouvrage intitulé Les antifeujs. Le livre blanc des violences antisémites en France depuis septembre 2000. Là encore, antisémitisme et antisionisme sont associés : "L'antisémitisme reculera le jour où l'antisionisme cessera de lui servir d'alibi. II n'est pas innocent de reprocher aux juifs leur solidarité avec Israël, ni de les obliger à se justifier d'être ce qu'ils sont pour les écarter de la communauté nationale. » (UEJF, SOS Racisme, 2002, p. 28) La rhétorique assimilant antisionisme et antisémitisme reçoit enfin une forme de caution officielle en 2004 dans le rapport remis par Jean-Christophe Rufin au ministre de l'Intérieur. L'auteur du rapport qualifie en effet l'« antisionisme radical» d'« antisémitisme par procuration » (Rufin, 2004, p. 28). Tout le problème réside dans l'appréciation du degré de "radicalité», car Jean-Christophe Rufin ne définit pas de critères permettant d'affirmer que certains discours antisionistes sont assimilables à de l'antisémitisme tandis que d'autres ne le sont pas.

Si une liste de critères précis n'est probablement pas définissable, il est toutefois intéressant de noter que les groupuscules qui tiennent un discours antisioniste flirtant avec l'antisémitisme sont condamnés par les principales associations pro-palestiniennes - comme l'Association France Palestine Solidarité (AFPS) - et rejetées en marge du mouvement de solidarité avec les Palestiniens. Des groupuscules comme La Pierre et l'Olivier, le Parti des musulmans de France ou le collectif cheikh Yassine sont ainsi considérés avec la plus grande circonspection par les associations pro-palestiniennes consensuelles. Quant à la «liste antisioniste » sur laquelle Dieudonné s'est présenté aux élections européennes de 2009, elle a été dénoncée par certaines des associations propalestiniennes les plus en vue, à l'instar de l'AFPS. Cette dernière a publié, quelques jours avant les élections, un communiqué dénonçant fermement l'engagement politique de l'humoriste mais dans lequel le mot "antisémitisme » n'était pas explicitement employé41.

Si la plupart des pro-palestiniens insistent sur le fait que l'antisionisme peut être légitime alors que l'antisémitisme doit être condamné, ils ne se disent pas pour autant antisionistes, soit parce qu'ils ne le sont pas, soit parce qu'ils

41. «La présentation par Dieudonné d’une liste prétendument antisioniste et constituée de militants d'extrême droite aux élections européennes mérite une mise au point ». Communiqué de l’AFPS publié le 26 mai 2009 [http:// www.france-palestine.org/article11922.html], consulté le 24 novembre 2009 . 
sentent que ce n'est pas une opinion consensuelle. Cela ressort nettement des entretiens réalisés avec des militants. Richard Wagman, militant d'extrême gauche et président de l'Union juive française pour la paix (UJFP) - une des rares associations juives à être présente dans les manifestations pro-palestiniennes -, souligne par exemple que la structure qu'il dirige «n'est pas officiellement antisioniste», avant d'ajouter : «Nous ne nous définissons pas comme une organisation sioniste dans la mesure où nous n'appuyons pas cette idée que les juifs du monde entier doivent nécessairement bénéficier d'un territoire ou de structures étatiques. ${ }^{42}$ Si les membres de cette association ne se perçoivent ni comme sionistes ni comme antisionistes, il est alors légitime de se demander comment ils se définissent. Certains éléments de réponse sont fournis par une autre cadre de l'UJFP, Michèle Sibony. Cette dernière rejette elle aussi le terme antisioniste 43 avant de qualifier l'association à laquelle elle appartient de «non-sioniste» ou d' «a-sioniste». Elle explique que le courant israélien qui se rapproche le plus de la philosophie de l'UJFP est le «post-sionisme »44. Richard Wagman et Michèle Sibony résument tous deux leur opinion en une phrase : «Il faut qu'lsraël devienne l’État de tous ses citoyens », ce qui signifie en clair que les non-juifs vivant en Israël ne se sentiront pas pleinement citoyens de cet État tant que ce dernier continuera à être défini officiellement comme un État juif.

Pour nombre de militants pro-israéliens, remettre en cause le caractère juif de l'État d'Israël ne relève ni de l' «a-sionisme » ni du «post-sionisme » mais bel et bien de l' "antisionisme» et donc, dans une certaine mesure, de l'antisémitisme. À ceux qui expliquent que traiter d'antisémites des juifs - à l'instar des membres de l'UJFP - ne fait pas sens, certains pro-israéliens rétorquent que les juifs antisémites existent et qu'ils souffrent d'un syndrome appelé la « haine de soi ». L'expression «haine de soi »-Selbsthaß, en allemand - a été popularisée par le philosophe Theodor Lessing (1872-1933)45. Au début de l'année 2009, l'Union des patrons et professionnels juifs de France (UPJF) a par exemple reproduit sur son site Internet un article d'Itshak Lurçat intitulé : «La haine de soi juive, du pathologique au politique »46. Dans cet article, l'Union juive française pour la Paix (UJFP) est qualifiée d' "association de juifs antisémites». La Paix Maintenant est aussi dans le collimateur de l'auteur :

La haine de soi est sans doute un facteur d'explication de l'attitude de groupes tels que Chalom Archav [La Paix maintenant], qui imputent toujours à Israël la responsabilité du conflit et des guerres imposées par nos ennemis arabes. Et c'est aussi

42. Entretien avec Richard Wagman réalisé par l'auteur le 13 avril 2006.

43. Entretien avec Michèle Sibony réalisé par l'auteur le 11 juillet 2007.

44. Sur le «post-sionisme», voir Nimni éd., 2003. Voir aussi Charbit, 2006.

45. Benoît, 1996, p. 213-222. Voir aussi la postface de Denis Charbit au livre d’Avraham B. Yehoshua, 2005 , p. 155.

46. Itshak Lurçat, "La haine de soi juive, du pathologique au politique », 30 janvier 2009 [http:// www.upjf.org/actualites-upjf/article-15622-145-7-haine-juive-pathologique-au-politiqueitshak-lurcat.html], consulté le 16 mai 2009. 
cette haine de soi pathologique qui explique le comportement délirant de certains "alterjuifs" en France (et ailleurs), dont nous avons eu plusieurs illustrations récentes, à l'occasion de la guerre contre le Hamas à Gaza.47

En définitive, cet article montre comment les tensions dans lesquelles se trouve pris un mot finissent par le rendre, si ce n'est inutilisable, du moins tellement connoté qu'il en devient à son tour source de discorde. Les différences de vues qui opposent militants pro-israéliens et pro-palestiniens sur la question de la définition du sionisme ne font en réalité qu'illustrer l'incompréhension fondamentale de deux sphères militantes qui interprètent le conflit israélopalestinien à travers des grilles de lecture antagonistes : celle du colonialisme pour les militants pro-palestiniens et celle du retour du peuple juif sur sa terre du côté pro-israélien.

Les militants des deux bords disposent de leurs propres médias, de leurs propres intellectuels et de leur propre littérature qui ne font que les renforcer dans leurvision du conflit plutôt que de les confronter à des visions alternatives. Lorsque des membres d'associations pro-palestiniennes et pro-israéliennes se rencontrent - ce qui est très rare -, ils se comprennent d'autant moins que les termes qu'ils emploient peuvent avoir des significations différentes en fonction de la sphère militante à laquelle ils appartiennent. En 1997, alors que l'euphorie des accords d'Oslo se faisait encore sentir, la revue Mots. Les langages du politique avait publié un numéro intitulé Israël, Palestine. Mots d'accord et de désaccord. Aujourd'hui, après une décennie marquée par la violence au Proche-Orient, les mots de désaccord semblent plus nombreux que jamais.

\section{Références}

Aвıтbol Michel, 2002, «Démocratie et religion en Israël », Cités, nº 4, p.15-32.

AlLALI Jean-Pierre, 2002, Les habits neufs de l'antisémitisme. Anatomie d'une angoisse, Paris, Desclée de Brouwer.

BÉNICHOU Delphine, 2008, Le sionisme dans les textes, Paris, CNRS.

BENoît Martine, 1996, "Theodor Lessing et le sionisme», Revue des études juives, vol. 155, n$^{0} 1-2$, p. 213-222.

BENSOUSSAN Georges, 2001, Une histoire intellectuelle et politique du sionisme (18601940), Paris, Fayard.

Brauman Rony, Finkielkraut Alain, 2006, La discorde. Israël-Palestine, les juifs, la France, Paris, Mille et Une Nuits.

Brenner Emmanuel éd., 2004 [2002], Les territoires perdus de la République. Antisémitisme, racisme et sexisme en milieu scolaire, Paris, Mille et Une Nuits.

Bernard Paul, 2003, "Sioniste et citoyen», Le sionisme expliqué à nos potes, Paris, La Martinière / UEJF.

47. Ibid. 
Bishara Marwan, 2002, Palestine-Israël. La paix ou l'apartheid, Paris, La Découverte. BONIFACE Pascal, 2003, Est-il permis de critiquer Israël?, Paris, Robert Laffont.

CARTER Jimmy, 2007, Palestine. La paix, pas l'apartheid, Paris, L'Archipel.

ChArbit Denis, 1998, Sionismes. Textes fondamentaux, Paris, Albin Michel.

- 2006, "Qu'est-ce qu'une nation post-sioniste?», Controverses, n³, octobre, p. 88-114.

- 2007, Qu'est-ce que le sionisme?, Paris, Albin Michel.

FIN KIELKRAUt Alain, 1983, La réprobation d'Israël, Paris, Denoël.

FISHMAN Joshua A., 1987, Ideology, Society and Language. The Odyssey of Nathan Birnbaum, Ann Arbor, Karoma.

GhILES-Meilhac Samuel, 2009, «De la discorde au fragile compromis. Le sionisme au cœur des débats lors de la création du Crif», Bulletin du centre de recherche français de Jérusalem, $\mathrm{n}^{\circ} 20$, en ligne [http://bcrfj.revues.org/index6178.html]. Consulté le 30 novembre 2010.

GoldNADEL Gilles W., 2001, Le nouveau bréviaire de la haine, Paris, Ramsay.

GREILSAMmer Ilan, 2005, Le sionisme, Paris, PUF.

HERzL Théodore, 2007 [1896], L'État juif, Paris, L'Herne (2008, L'État des juifs, Paris, La Découverte).

Hess Moses, 1981 [1862], Rome et Jérusalem, Paris, Albin Michel.

KüHNTOPF-Gentz Michael, 1992, " “Israel geht vor Sion”. Nathan Birnbaum und die Palästinafrage », Zeitschrift für Religions- und Geistesgeschichte, vol. XLIV, n 2, p. 118-139.

KLugman Patrick, 2003, "En guise de postface. Sioniste et pro-palestinien», Le sionisme expliquéà nos potes, Paris, La Martinière / UEJF, p. 243-253.

LAQUEUR Walter, 1973, Histoire du sionisme, Paris, Calmann-Lévy.

Liebman Marcel, 1970, «Antisémitisme et antisionisme. L'impossible amalgame», Mai, nº 10 , février.

Lustman François, LÉvy Monique, Toublanc Stéphane, 2004, «Résister à l'emprise du Consistoire à Paris dans la première moitié du XIxe siècle. La synagogue Sauphar», Archives juives, nº 2, p. 104-110.

NiCAULT Catherine, 2001, "L'Alliance au lendemain de la seconde guerre mondiale. Ruptures et continuités idéologiques », Archives juives, nº 1, p. 23-53.

NımNı Ephraïm éd., 2003, The Challenge of Post-Zionism. Alternatives to Israeli Fundamentalist Politics, Londres, Zed Books.

PINSKER Léon, 2006 [1882], Autoémancipation! Avertissement d'un juif russe à ses frères, Paris, Mille et Une Nuits.

Poliakov Léon, 1951, Bréviaire de la haine. Le IIle Reich et les Juifs, Paris, Calmann-Lévy.

- 1969, De l'antisionisme à l'antisémitisme, Paris, Calmann-Lévy.

RAVEnEL Bernard, 2007, «Dans la revue des Temps Modernes : le basculement conceptuel de Maxime Rodinson» [http://www.france-palestine.org/article6719.html], 28 septembre. Consulté le 14 mars 2011.

Rodinson Maxime, 1967, «Israël, fait colonial?», Les Temps modernes, n² 253 bis, p. $17-88$.

RUFIN Jean-Christophe, 2004, Chantier sur la lutte contre le racisme et l'antisémitisme. Rapport remis au ministre de l'Intérieur, Paris, La Documentation française. 
SARFATI Georges E., "La charte de l'OLP en instance d'abrogation », Mots. Les langages du politique, $\mathrm{n}^{\circ}$ 50, mars 1997, p. 23-39.

Sieffert Denis, 2003, "Antisémitisme, entre réalités et manipulations», Antisémitisme : l'intolérable chantage? Israël-Palestine, une affaire française, E. Balibar, R. Brauman, J. Butler et al. éd., Paris, La Découverte.

TAgUiEfF Pierre-André, 2002, La nouvelle judéophobie, Paris, Mille et Une Nuits.

UNION DES ÉTUDIANTS JUIFS DE FRANCE, SOS RACISME, 2002, Les antifeujs. Le Livre blanc des violences antisémites en France depuis septembre 2000, Paris, Calmann-Lévy.

Wieviorka Michel éd., 2005, La tentation antisémite. Haine des juifs dans la France d'aujourd'hui, Paris, Robert Laffont.

Yehoshua Avraham B., 2005, Israël, un examen moral, Paris, Calmann-Lévy. 